\title{
Neurophysiological observations on corticospinal projections to the upper limb in subjects with Rett syndrome
}

\author{
J A Eyre, A M Kerr, S Miller, M C O'Sullivan, V Ramesh
}

\begin{abstract}
The aim of the present study was to investigate the excitability of corticospinal neurons and the integrity of their projections to the alpha motor neurons through the corticospinal tract in subjects of different ages with Rett syndrome. Electromagnetic stimulation of the motor cortex and cervical motor roots was used to evoke motor action potentials in the biceps brachii and hypothenar muscles. The phasic stretch refiex in the biceps brachii was also recorded to study the excitability of spinal alpha motor neurons. Motor cortex stimulation evoked motor action potentials at low threshold and with abnormally short latencies and prolonged durations. In contrast cervical motor root stimulation resulted in responses of normal latency and duration. The phasic stretch reflex had a low threshold, short latency and prolonged duration. It is concluded that in Rett syndrome the corticospinal pathway is intact. The results suggest disordered synaptic control of the Betz cell of the motor cortex and/or the spinal alpha motor neuron, although the involvement of the latter might be a consequence of dysfunction in supraspinal descending motor pathways.
\end{abstract}

University of

Newcastle upon Tyne

Department of Child

Health

J A Eyre

M C O'Sullivan

$\checkmark$ Ramesh

School of

Neuroscience

$S$ Miller

Department of Child

Health, Glasgow

University and

Monitoring Unit,

Quarrier's Homes,

Bridge of Weir,

Scotland

A M Kerr

Correspondence to: Dr Eyre, Paediatric Neurology

Paediatric Neurol:

Research Group,

Department of Child

The Medical School,

Newcastle upon Tyne

University, Newcastle upo

Tyne NE2
Kingdom.

Received 31 August 1989 and in revised form 13 February

1990

Accepted 7 March 1990
Severely disordered control of movement associated with profound mental handicap is the hallmark of Rett syndrome $\mathrm{e}^{1-3}$ a neurological disorder which exclusively affects females. The motor component of the syndrome is highly characteristic and includes abnormal control of voluntary movement, disordered tone, spasticity and the presence of involuntary movements. ${ }^{4}$

Rett syndrome affects one in 12000 females, ${ }^{56}$ the signs appearing in the first year after birth, while development is apparently normal. A period of regression of one to two years then follows, when skills in speech, manipulation and locomotion are lost..$^{1-7}$ The girls with Rett syndrome are then left with paucity of voluntary movement, poor coordination, and jerking and writhing involuntary movements, which are most marked in the hands and arms but also affect the lower limbs, trunk and face. ${ }^{1-3}$

Following the regression period purposeful hand use is particularly impaired with lack of fine finger control. The tendon reflexes are brisk, ankle clonus is frequently present; the
Babinski sign has been reported to be positive in some subjects ${ }^{1-3}$ but not in others. ${ }^{6}$ Muscle tone tends to increase with age and this is usually associated with spasticity. ${ }^{1-3}$ Taken together these signs suggest involvement of corticospinal neurons projecting from the motor cortex in the pathophysiology of the movement disorder.

The aim of this study was therefore to use the non-invasive method of electromagnetic stimulation of the motor cortex to investigate the excitability of corticospinal neurons and the integrity of their projections to alpha motoneurons through the corticospinal tract in subjects of different ages with Rett syndrome. The phasic stretch reflex was also recorded to study the excitability of spinal alpha motor neurons.

\section{Methods}

Subjects

The study was performed in eight subjects, between five and 26 years, who fulfilled all the necessary criteria and many of the supportive criteria for a diagnosis of Rett syndrome, as specified by the Rett syndrome diagnostic criteria work group. ${ }^{4}$ The clinical details are summarised in table 1 . The neurophysiological data obtained from these subjects were compared with those of 350 normal subjects aged from birth to adulthood. ${ }^{1011}$ Approval of the University of Newcastle upon Tyne Ethical Committee and informed parental consent were obtained.

\section{Electromyograms}

Electromyograms were recorded with surface mounted, standard EEG electrodes placed on the belly of the right biceps brachii and over the right hypothenar muscles. The signals were amplified by a Nicolet amplifier (CA $1000)$ and filtered with a bandpass $-3 \mathrm{~dB}$ at 5 $\mathrm{Hz}$ and $1.5 \mathrm{kHz}$.

Electromagnetic stimulation of the motor cortex In all subjects the motor cortex was excited with an electromagnetic stimulator (MagStim 200, Novametrix). The stimulating coil (diameter $9 \mathrm{~cm}$ ) was held over the scalp, tangential to the vault of the skull, and was positioned to obtain motor action potentials in the biceps brachii and hypothenar muscles.

In the normal subjects motor action potentials were evoked initially when both muscles were contracting. Muscle contraction was obtained in those subjects older than two years by placing a weight in the out-stretched, 
Table 1

\begin{tabular}{lrrrrrrrr}
\hline Subject & 1 & 2 & 3 & 4 & 5 & 6 & 7 & 8 \\
\hline Age (years) & 5 & 7 & 8 & 8 & 8 & 9 & 16 & 26 \\
Height (cm) & 111 & 108 & 121 & 121 & 111 & 116 & 136 & 152 \\
$\begin{array}{l}\text { Arm length C, to fifth finger } \\
\quad \text { tip (cm) }\end{array}$ & 59 & 55 & 66 & 64 & 60 & 63 & 78 & 75 \\
$\begin{array}{l}\text { Length C5 to midpoint } \\
\quad \text { biceps brachii (cm) }\end{array}$ & 19 & 18 & 22 & 21 & 20 & 21 & 26 & 25 \\
Scoliosis & + & + & + & + & + & + & + & + \\
Hyperventilation & + & + & + & + & + & + & + & + \\
Walking independently & + & - & - & + & + & + & + & + \\
Increased biceps reflexes & + & + & + & + & + & + & + & + \\
Increased patellar reflexes & + & + & + & + & + & + & + & + \\
Increased ankle reflexes & + & + & + & + & + & + & + & + \\
Ankle clonus & + & + & + & + & + & - & - & + \\
Babinski sign & + & - & - & - & - & - & - & + \\
Spasticity & + & + & + & + & + & + & + & + \\
\hline
\end{tabular}

Definitions

$+=$ present

$+=$ present
$-=$ not present

Hyperventilation: intermittent hyperventilation and breath-holding.

Spasticity: velocity dependent increase of muscle tone following stretch. ${ }^{25}$

supinated hand, with the arm adducted at the shoulder and flexed at the elbow to about $90^{\circ}$. In those aged less than two years muscle contraction was elicited using the traction reflex. An attempt was then made to evoke motor action potentials in the muscles when relaxed.

The subjects with Rett syndrome were not able to hold a weight in their hand nor were they able to sustain a contraction voluntarily. In these subjects the evoked motor action potentials were only studied in relaxed muscles.

\section{Electromagnetic stimulation of the cervical motor roots}

In all normal subjects and in the five subjects with Rett syndrome that were tested (subjects $1,2,3,4,5)$ the cervical motor roots were stimulated electromagnetically by placing the stimulating coil (diameter $7 \mathrm{~cm}$ ) in the coronal plane dorsal to the cervical spines $C_{5}$ to $C_{8}$. The coil position was adjusted and the stimulus intensity reduced to obtain the longest latency to the onset of motor action potentials in the relaxed biceps brachii and the hypothenar muscles. ${ }^{12}$

Phasic stretch reflex

The phasic stretch reflex in the biceps brachii was elicited with a hand held electro-mechan- ical vibrator (model 201, Ling Altec) delivering a single cycle of a $150 \mathrm{~Hz}$ sine wave, peak to peak amplitude $0.5 \mathrm{~mm}$. The stimulus started from the zero crossing point of the sine wave with an outward movement. The stylus of the vibrator was placed over the tendon of the biceps brachii in the cubital fossa. In each normal subject an attempt was made to obtain a phasic stretch reflex first in the relaxed and then in the contracting biceps brachii. In the subjects with Rett syndrome the reflex was only studied in relaxed muscle.

\section{Data analysis}

For Rett cases and normal subjects the motor action potentials evoked by electromagnetic stimulation and vibration were recorded on magnetic tape (Racal Store 4 Tape Recorder) and analysed off-line. Following cortical stimulation eight motor action potentials in both the biceps brachii and hypothenar muscles were recorded. The latency was determined as the shortest latency in any single response; the duration of the response was determined from the average of the eight responses. Following cervical spine stimulation the latency was determined from the longest latency of any single response and the duration from the average of eight responses. For the stretch reflex the onset latency was determined by the shortest latency of any single response and the duration was measured from averages of 20 motor action potentials.

\section{Results}

1) Electromagnetic stimulation of motor cortex and cervical motor roots

In the eight subjects with Rett syndrome electromagnetic stimulation of the motor cortex evoked motor action potentials in the biceps brachii and hypothenar muscles. Figure 1 shows representative recordings of evoked motor action potentials in the biceps brachii and hypothenar muscles in subject 3 aged eight years and from an eight year old normal subject. It is important to note that in normal subjects below the age of nine years electromagnetic stimulation of the motor cortex

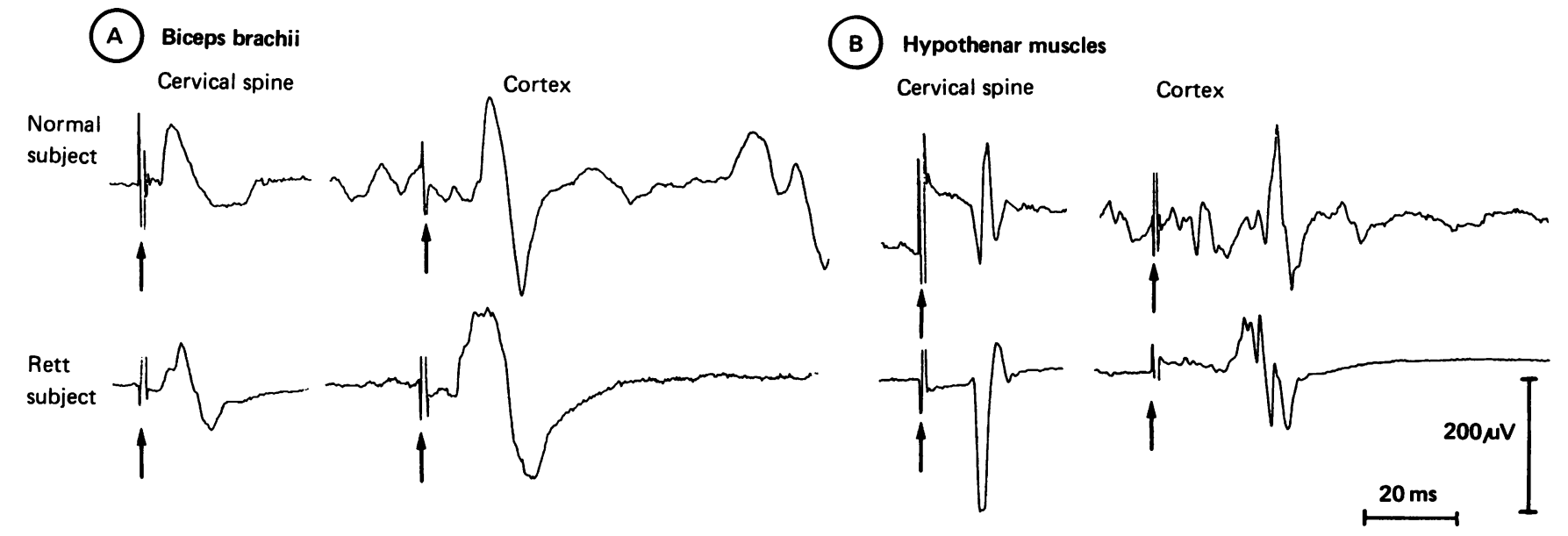

Figure 1 Motor action potentials following electromagnetic stimulation of cervical spine and motor cortex. Arrows indicate timing of stinulation. 
Figure 2 Onset latencies of motor action potentials following electromagnetic stimulation of the motor cortex in relation to age of subjects. The filled and open circles indicate the median values for normal subjects in contracted and relaxed muscles,

respectively. The hatched area defines for normal subjects the interquartile range in contracted muscle and the stippled area the interquartile range in relaxed muscle. The triangles indicate data for the Rett subjects in relaxed muscle.
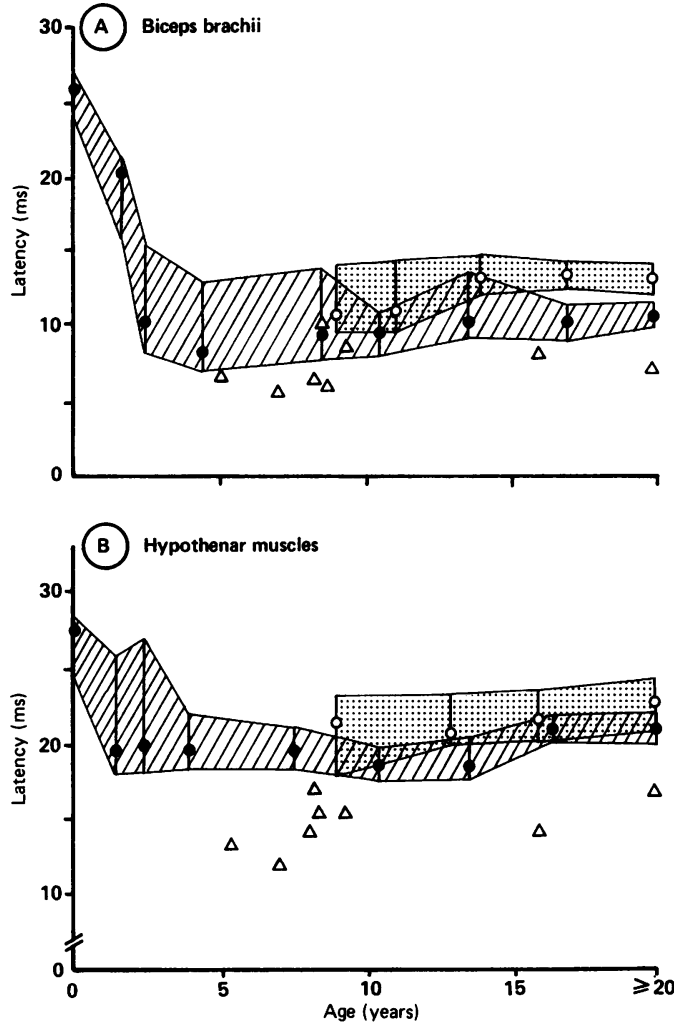

does not excite motor action potentials in relaxed muscles even when $100 \%$ of stimulator output is used; facilitation by background muscle contraction is required for a response $\mathrm{e}^{10}$ (fig 1). In all eight subjects with Rett syndrome, including the five subjects aged less than nine years, motor action potentials could easily be evoked in both the biceps and hypothenar muscles when they were relaxed, indicating a lower threshold for activation (fig 1).

In figs $2 A$ and $B$ the latencies of onset of the evoked motor action potentials in the relaxed biceps brachii and hypothenar muscles follow- ing brain stimulation are shown in relation to the normal latencies for age in both contracted and relaxed muscles. The latencies for the eight Rett subjects are below the lower interquartile range for age in both muscles, when relaxed. In the hypothenar muscles the onset latencies for all subjects also lie below the normal values for both contracted and relaxed muscle. In the biceps the values for two subjects lie within the interquartile range for contracted muscle. The low values of these latencies might be attributable to a shorter conduction pathway in view of the subjects' short stature (table 1). In fig 3A therefore the latencies to the biceps are plotted in relation to the distance from $\mathrm{C}_{5}$ spine to the mid-point of the biceps brachii. Similarly, in fig 3B the latencies to the hypothenar muscles are plotted against the length of the arm $\left(\mathrm{C}_{5}\right.$ spine to tip of the fifth finger). The values for the Rett subjects still lie below the lower interquartile range for relaxed muscle indicating a real shortening of the latency.

The durations of the motor action potentials evoked in relaxed biceps and hypothenar muscles in the Rett subjects are shown in fig $4 \mathrm{~A}$ and $B$ in relation to data from normal subjects for both relaxed and contracted muscles. In both muscle groups the durations of the evoked motor action potentials lie well above the normal range with some values more than twice the upper limit of the interquartile range.

In the five Rett subjects in whom cervical motor root stimulation was studied, the motor action potentials in both the biceps brachii and the hypothenar muscles were of normal onset latency and duration for age (fig 1).

\section{2) Phasic stretch reflex}

In the eight Rett subjects phasic stretch reflexes were evoked in relaxed biceps brachii following mechanical vibration of the tendon. Figure 5 shows representative recordings of motor action potentials evoked in subject 3 and

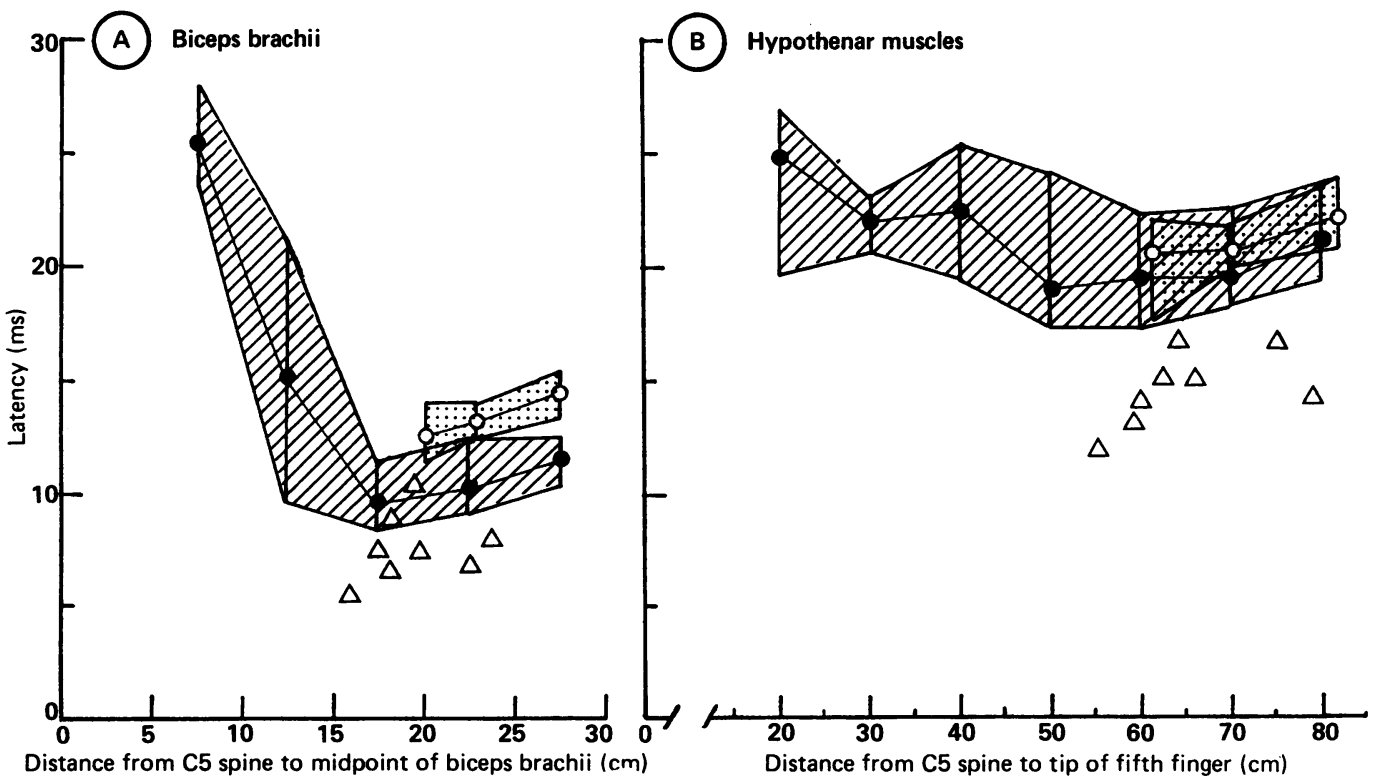

Figure 3 Onset latencies of motor action potentials following electromagnetic stimulation of motor cortex in relation to conduction distance. The filled and open circles indicate the median values for normal subjects in contracted and relaxed muscles, respectively. The hatched area defines for normal subjects the interquartile range in contracted muscle and the stippled area the interquartile range in relaxed muscle. The triangles indicate data for the Rett subjects in relaxed muscle. 
Figure 4 Durations of motor action potentials following electromagnetic stimulation of motor cortex in relation to age of subjects. The filled and open circles indicate the median values for normal subjects in contracted and relaxed muscles,

respectively. The hatched area defines for normal subjects the interquartile range in contracted muscle and the stippled area the interquartile range in relaxed muscle. The triangles indicate data for the Rett subjects in relaxed muscle.

Figure 5 Recordings of phasic stretch reflexes in a normal subject and a Rett subject, both of eight years of age. Arrows indicate timing of stimulus. in an aged matched normal subject. It should be noted that in normal subjects above the age of two years it is not possible to evoke the reflex in relaxed muscle using this stimulus; a background muscle contraction is required to obtain a response. ${ }^{11}$ In the eight Rett subjects, all older than two years, phasic stretch reflexes could be elicited in relaxed biceps, indicating a lower threshold of activation.

The latency of onset of the phasic stretch reflex in relation to the ages of the Rett subjects is shown in comparison to data obtained from normal subjects in fig 6 . The latencies obtained from the Rett subjects tended to be short, with the data from seven subjects clustering about or below the lower interquartile range for normal
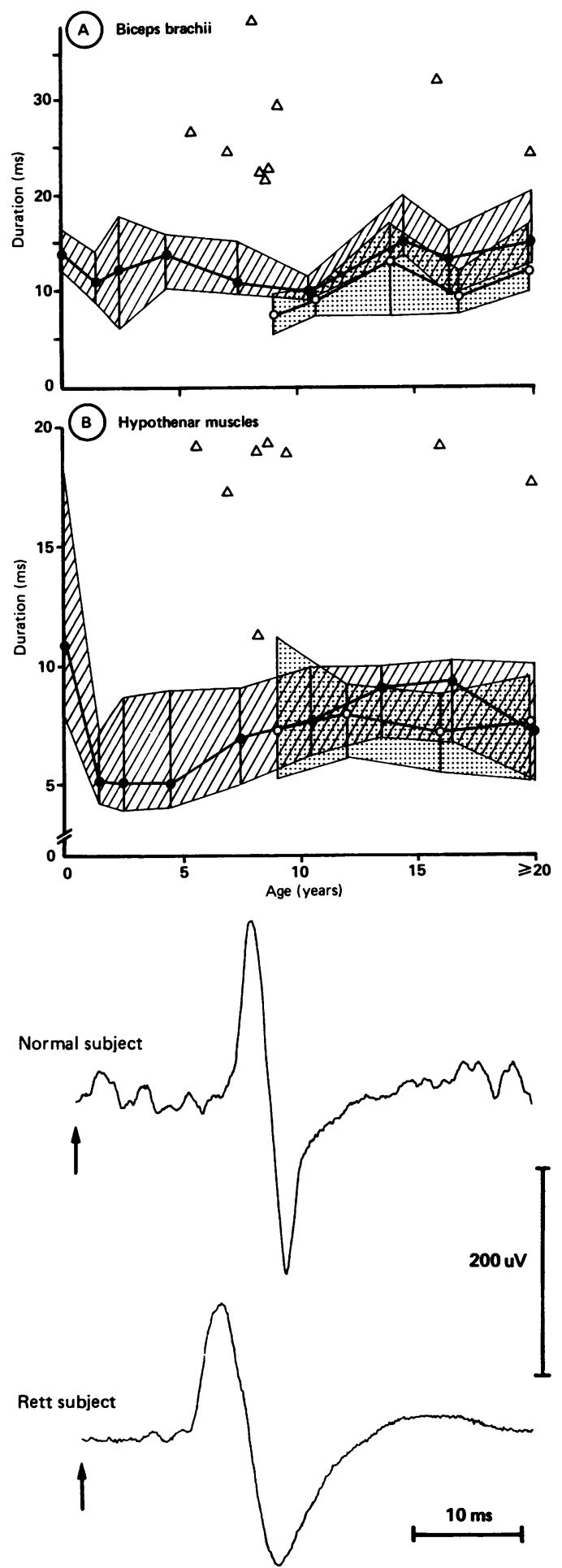

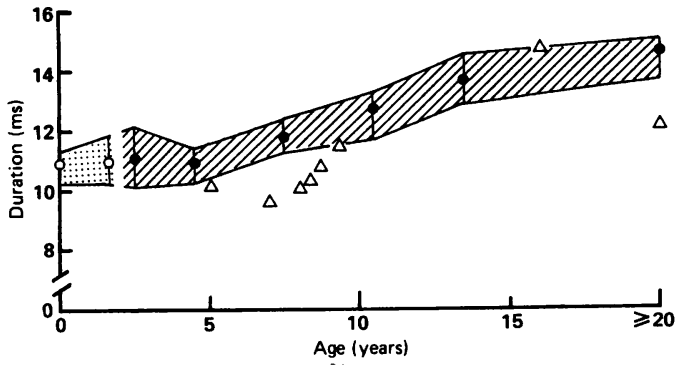

Figure 6 Onset latencies of phasic stretch reflexes in the biceps brachii in relation to age of subjects. The filled and open circles indicate the median values for normal subjects in contracted and relaxed muscles, respectively. The hatched area defines for normal subjects the interquartile range in contracted muscle and the stippled area the interquartile range in relaxed muscle. The triangles indicate data for the Rett subjects in relaxed muscle.

subjects in contracting muscle. As in fig $3 \mathrm{~A}$ and $B$, a plot of the latencies in relation to arm length still showed the latencies to be short for six of the Rett subjects (fig 7). The latency of the phasic stretch reflex in the Rett subjects is obtained from relaxed muscle and that from the normal subjects when the biceps brachii was contracting. If it were possible to elicit the stretch reflex in normal subjects in relaxed muscles the onset latency would be longer than that in contracting muscle because of the need

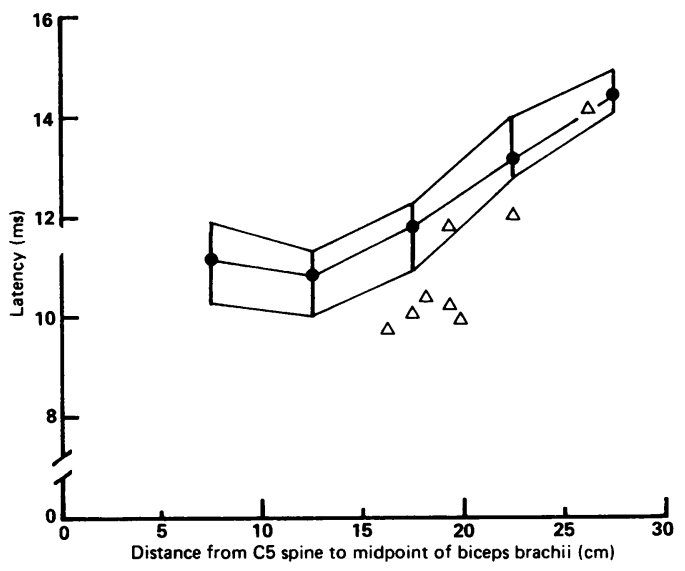

Figure 7 Onset latencies of phasic stretch reflexes in biceps brachii in relation to conduction distance. The filled circles indicate the median values for normal subjects. The open area defines for normal subjects the interquartile range. The data have not been plotted with respect to contracted and relaxed muscle, because of the overlap of arm length up to the age of four years.

However, all the data relating to distances above $10 \mathrm{~cm}$ relate to contracted muscle. The triangles indicate data of the Rett subjects in relaxed muscle.

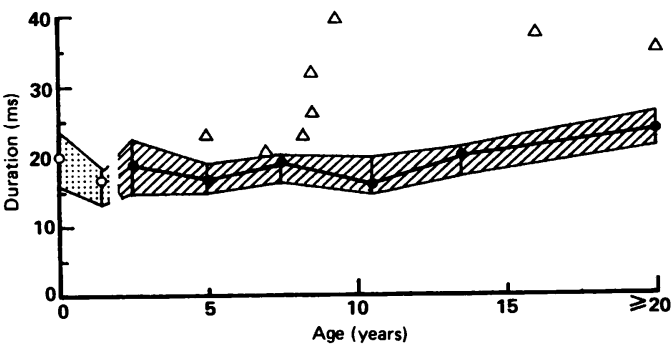

Figure 8 Durations of phasic stretch reflexes in biceps brachii in relation to age of subjects. The filled and open circles indicate the median values for normal subjects in contracted and relaxed muscles, respectively. The hatched area defines for normal subjects the interquartile range in contracted muscle and the stippled area the interquartile range in relaxed muscle. The triangles indicate data for the Rett subjects in relaxed muscle. 
for greater temporal summation of the afferent excitatory input to the motor neuron. Thus a comparison of the latency in relaxed muscle in the Rett subjects with normal data from contracting muscles is likely to underestimate any degree of shortening of the onset latency.

The durations of the phasic stretch reflexes in the Rett subjects were longer than in normal subjects (fig 8).

\section{Discussion}

In the subjects with Rett syndrome motor action potentials could be evoked easily following electromagnetic stimulation of the motor cortex. This was unexpected in view of the severe impairment of voluntary movement control and the clinical signs, both of which suggest pathology of the corticospinal pathway.

Studies in monkeys have shown that electromagnetic stimulation of the brain evokes both direct and indirect volleys ( $D$ and I waves) in the corticospinal tract. ${ }^{1314}$ In studies of adult human subjects there is evidence that electromagnetic stimulation excites the Betz cells in the motor cortex largely trans-synaptically. ${ }^{15-17}$ Therefore, the ability to evoke motor action potentials following brain stimulation in Rett subjects may imply not only integrity of the corticospinal tract, but also the existence of functionally active, excitatory synaptic inputs to Betz cells in the motor cortex.

This study reports for the first time the occurrence of abnormally short latencies for the onset of motor action potentials following brain stimulation, in conjunction with abnormally prolonged durations and reduced thresholds for activation. The short latency indicates that either the largest myelinated fibres of the corticospinal tract conduct with faster velocities than normal or that the time for bringing the corticospinal neuron and/or the spinal alpha motor neuron to firing threshold is diminished.

An increased excitability of Betz cells and/or a greater density of excitatory inputs to these cells would result in a lower threshold for direct activation ( $\mathrm{D}$ waves) and a greater probability for evoking multiple I waves, leading to short latency and prolonged duration responses. The low threshold and the prolongation of the biceps motor response to both stretch and brain stimulation would also be predicted, if at the spinal level there were increased excitability and/or decreased inhibitory control of the alpha motor neuron. This proposal is supported by the observation that the motor action potentials evoked by stretch of biceps brachii have similar characteristics to those following brain stimulation: short latencies in comparison to age matched normal subjects, prolonged duration of response and low threshold for activation. ${ }^{11}$ The conduction delays of the peripheral efferent and afferent components of the phasic stretch reflex are likely to be normal, as evidenced by 1) the normal onset latencies and durations of the motor action potentials following electromagnetic stimulation of cervical motor roots in the five Rett subjects examined in this study, and 2) the normal durations and the normal or even slightly prolonged latencies of the peripheral components of the somatosensory evoked potential observed in subjects with Rett syndrome. ${ }^{18} 19$ The short latencies of the phasic stretch reflex are therefore likely to result from a reduction in the synaptic delay and the prolonged responses from prolonged alpha motor neuron activation.

Although neuropathological studies have reported some gross abnormalities in subjects with Rett syndrome, for example, decreased brain weight, cortical atrophy and some slight atrophy of spinal grey matter, ${ }^{2021}$ no unequivocally specific changes have been demonstrated. The most striking neurophysiological abnormalities so far documented concern the wide-spread and severe abnormality of the EEG observed in all subjects tested ${ }^{22}{ }^{23}$ suggesting diffuse, bilateral cortical involvement. The virtual absence of voluntary movement, particularly of the hand and fingers, together with the observations in this study of normal or possibly faster than normal conduction in the corticospinal pathway, provides further evidence that the disorder lies "upstream" of the outflow from the motor cortex. Thus the lesion may involve motor and/or sensory association areas, or the systems concerned with motor planning which project to the motor cortex, such as cerebellum, basal ganglia and limbic lobe. Our results also suggest disordered synaptic control of the Betz cell of the motor cortex and/or the spinal alpha motor neuron, although the involvement of the latter might be a consequence of dysfunction in supraspinal descending control.

We gratefully acknowledge the participation of the subjects with Rett syndrome and their families. Financial support was provided by the Quarrier's Homes, Bridge of Weir, Scotland, National Rett Syndrome Association, United Kingdom Rett Syndrome Association (Scotland), Medical Research Council, Spastics Society, and Wellcome Trust.

1 Rett A. Uber ein eigenartiges hirnatrophisches Syndrom bein Hyperammoniämir in Kindesalter. Wien Medische Wochenschriff 1966;116:723-38.

2 Rett A. Cerebral atrophy associated with hyperammonaemia. In: Vinken PJ, Bruyn GW, ed. Handbook of clinical

3 Hagberg B, Aicardi J, Dias K, Ramos O. A progressive syndrome of autism, dementia, ataxia, and loss of purposeful hand use in girls: Rett's syndrome: Report of 35 cases. Ann Neurol 1983;14:471-9.

4 The Rett Syndrome Diagnostic Criteria Work Group. Diagnostic criteria for Rett Syndrome. Ann Neurol 1988;23:425-8.

5 Kerr AM, Stephenson JBP. A study of the natural history of Rett syndrome in 23 girls. Am JMed Genet 1986;24:77-83

Kerr AM, Stephenson JBP. Rett's syndrome in the West of Scotland. Br Med J 1985;291:579-82.

7 Kerr AM, Montagu J, Stephenson JBP. The hands, and the mind, pre- and post-regression in Rett syndrome. Brain mind, pre- and post

8 Barker AT, Freeston IL, Jalinous R, Jarratt JA. Magnetic stimulation of the human brain and peripheral nervous system: an introduction and results of an initial clinical evaluation. Neurosurgery 1987;20:100-9.

9 Mills K, Murray NMF, Hess CW. Magnetic and electrical transcranial brain stimulation: physiological mechanism and clinical applications. Neurosurgery 1987;20:164-8.

10 Koh THHG, Eyre J. Maturation of corticospinal tracts studied by electromagnetic stimulation of the motor cortex. Arch Dis Child 1988;63:1347-52.

11 Eyre JA, Miller S, O'Sullivan MC, Ramesh V. Radiation of the phasic stretch reflex in biceps brachii to muscles of the arm and shoulder in the human neonate, and its restriction with increasing age. J Physiol (London) Proc 1989. (In press).

12 Plassman BL, Gandevia SC. High voltage stimulation over the human spinal cord: sources of latency variation. $J$ Neurol Neurosurgery Psychiatry 1989;52:213-7.

13 Amassian VE, Quirk GJ, Stewart M. Magnetic coil versus 
electrical stimulation of monkey motor cortex. $J$ Physiol (London) 1987;394:119.

14 Edgley SA, Eyre JA, Lemon RN, Miller S. Excitation of the corticospinal tract by electromagnetic and electrical stimulation of the scalp in the Macque monkey. J Physiol 1990;425:301-20.

15 Rothwell JC, Day BL, Thompson PD, Dick JPL, Marsden $\mathrm{CD}$. Some experiences of techniques for stimulation of the human cerebral motor cortex through the scalp. Neurosurgery 1987;20:156-63.

16 Boniface SJ, Mills KR, Schubert M. Multimodal discharge latencies of human spinal motoneurones evoked by transcranial magnetic stimulation. J Physiol (London) Proc 1989 (in press).

17 Day BL, Dressler D, Maartens de Noordhout A, Marsden CD, Nakashima K, Rothwell JC, Thompson PD. Electrical and magnetic stimulation of human motor cortex: trical and magnetic stimulation of human motor cortex: surface EMG and single mot
(London) $1989 ; 412: 449-73$

18 Verma NP, Nigro MA, Hart ZH. Rett syndrome-a gray matter disease? Electrophysiological evidence. Electroencephalogr Clin Neurophysiol 1987;67:327-9.
19 Bahr GG, Witt-Engerstrom I, Hagbert B. Brainstem and spinal cord impairment in Rett syndrome: Somatosensory
and auditory evoked responses investigations. Brain Dev and auditory ev

20 Jellinger K, Seitelberger F. Neuropathology of Rett Syndrome. Am Journal Med Genet 1986;24:259-88.

21 Oldfors A, Hagberg B, Nordgren H, Sourander P, WittEngerstrom I. Rett syndrome: Spinal cord neuropathology. Paediatric Neurology 1988;4:172-4.

22 Verma NP, Cheda RL, Ngro MA, Hart ZH. Electroencephalographic findings in Rett Syndrome. Electroencephalogr Clin Neurophysiol 1986;64:394-401.

23 Ho HH, Wong PK, Roberton R. Rett syndrome: Clinical profile and EEG abnormalities. Clin Invest Med 1988;11:234-41.

24 Southall DP, Kerr AM, Tirosh E, Amos P, Lang MH and Stephenson JBP. Hyperventilation in the awake state: Stephenson JBP. Hyperventilation in the awake state: potentially treatable compo
Dis Child 1988;63:1039-48.

25 Lance JW. Symposium synopsis. In: Feldman RG, Young RR, Koella WP, eds. Spasticity: disordered motor control. Chicago: Year Book Medical Publishers, 1980:485-94. 